SOI: $1.1 /$ TAS DOI: $10.15863 /$ TAS

\section{International Scientific Journal Theoretical \& Applied Science}

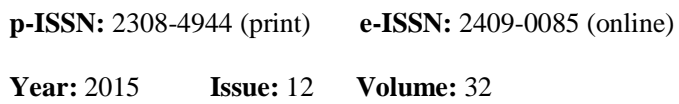

p-ISSN: 2308-4944 (print) e-ISSN: 2409-0085 (online)

Year: 2015 Issue: 12 Volume: 32

Published: $30.12 .2015 \quad$ http://T-Science.org

SECTION 4. Computer science, computer engineering and automation.
Telman Aliev

Director, doctor of technical Sciences, Professor, Institute of Control Systems of The National Academy of Sciences of Azerbaijan, Azerbaijan cavan62@mail.ru

Asif Rzaev

head of laboratory, candidate of technical Sciences, associate Professor, Institute of Control Systems of The National Academy of Sciences of Azerbaijan, Azerbaijan

Firudin Mamedov head of the Department of electrical engineering, Ph. D., Professor, Sumgayit State University, Azerbaijan

Javanshir Mamedov Professor of the Department "Information technologies and programming", Ph. D., Professor, Sumgayit State University, Azerbaijan

Khafiz Rasulov assistant of Professor, Department "Electromechanics", Sumgayit State University, Azerbaijan

\title{
DEVELOPMENT OF A SYSTEM OF MANAGEMENT AND CONTROL OF THE TECHNOLOGICAL PROCESS OF DRILLING OIL WELLS
}

Abstract: The article discusses the development of the control system and process control downhole pump in oil wells. The proposed integrated scheme for automated management and control of the technological process of drilling of oil wells in a sub-level of technical, software and information support. Developed a force transducer with cylindrical sensitive element for fixation of loads from rods deep pump into drill installation.

Depending on the requirements on the choice of material, structural dimensions and maximum loads the rod deep pump selects the microprocessor type ATmega32-16 and a block diagram of a control system of an oil well.

Key words: control system, sensor, rod, oil craft, well, rocking.

Language: Russian

Citation: Aliev T, Rzaev A, Mamedov F, Mamedov J, Rasulov K (2015) DEVELOPMENT OF A SYSTEM OF MANAGEMENT AND CONTROL OF THE TECHNOLOGICAL PROCESS OF DRILLING OIL WELLS. ISJ Theoretical \& Applied Science 12 (32): 108-112.

Soi: http://s-o-i.org/1.1/TAS-12-32-21 Doi: crossef http://dx.doi.org/10.15863/TAS.2015.12.32.21

\section{РАЗРАБОТКА СИСТЕМЫ УПРАВЛЕНИЯ И КОНТРОЛЯ ТЕХНОЛОГИЧЕСКОГО ПРОЦЕССА БУРЕНИЯ НЕФТЕДОБЫВАЮЩЕЙ СКВАЖИНЫ}

Аннотация: В статье рассматривается вопрос разработки системы управления и контроля технологического процесса глубинного насоса в нефтедобывающей скважине. Предложена комплексная автоматизированная схема для управления и контроля технологического процесса бурения нефтяных скважин на подуровнях технического, программного и информационного обеспечения. Разработан датчик усилия с чувствительным цилиндрическим элементом для фиксирования нагрузок от итанги глубинного насоса в бурильной установке.

В зависимости от требований по выбору материала изготовления, конструкционных размеров и максимальных нагрузок штанги глубинного насоса выбирается микропрочессор типа ATтеgа32-16 и структурная схема системы управления нефтяной скважины.

Ключевые слова: система управления, датчик, итанга, нефтедобывающий промысел, скважина, качалка. 


\begin{tabular}{|c|c|c|c|c|c|c|}
\hline Impact Factor: & $\begin{array}{l}\text { ISRA (India) } \\
\text { ISI (Dubai, UAF } \\
\text { GIF (Australia) } \\
\text { JIF }\end{array}$ & $\begin{array}{l}=1.344 \\
=0.829 \\
=0.564 \\
=1.500\end{array}$ & $\begin{array}{l}\text { SIS (USA) } \\
\text { PИНЦ (Russia } \\
\text { ESJI (KZ) } \\
\text { SJIF (Morocco }\end{array}$ & $\begin{aligned}= & 0.912 \\
= & 0.179 \\
= & \mathbf{1 . 0 4 2} \\
& =2.031\end{aligned}$ & $\begin{array}{l}\text { ICV (Poland) } \\
\text { PIF (India) }\end{array}$ & $\begin{array}{l}=6.630 \\
=1.940\end{array}$ \\
\hline
\end{tabular}

\section{Введение}

В настоящее время для обеспечения эффективной добычи нефти и долговременного функционирования нефтедобывающих установок с минимальными экономическими потерями следует решить ряд научно-инженерных задач по интеллектуализации уровня управления технологических операций бурильных скважин $[3,4]$ :

1. Создание комплексной схемы автоматизации для гибкого управления процессом нефтедобычи в скважинах с учетом воздействий внутренних технологических и внешних метереологических факторов;

2. Выбор технических устройств управления и контроля функций глубинного насоса нефтяной скважины [5];

3. Разработка нестандартных элементов для обеспечения работы интеллектуальной системы диагностирования нефтяной скважины.

\section{Решение}

Решение вопроса создания комплексной схемы автоматизации для гибкого управления процессом нефтедобычи в глубинных скважинах является поэтапным процессом [9]. На начальном этапе проводится технологический анализ нефтедобычи в глубинных скважинах. Технологические операции при работе качалки скважины характеризуются параметрами внутренних и внешних воздействий, таких как изменяющие силы в результате деформации штанги глубинного насоса, изменение температурного режима, а также нелинейным перемещением качалки. В соответствии с технологическими операциями выбираются типы датчиков фиксирования температурного режима, силы, возникающие от деформации штанги глубинного насоса, и датчиков нелинейного перемещения качалки для управления процессом добычи нефти. Обработка и управление данными от датчиков осуществляются с помощью микропроцессорной системы, установленная в автоматизированном рабочем месте с техническими средствами оперативного управления и контроля нефтедобывающей скважины, а также визуального представления выходных данных температурного режима окружающей среды, силы деформации при работе штанги скважины и объема добываемой нефти. [1]. Комплексная автоматизированная схема для управления и контроля технологического процесса бурения нефтяных скважин (ТПБНС) строится на подуровнях технического, программного и информационного обеспечения (рис. 1).

В информационной подсистеме комплексной системы управления и контроля ТПБНС хранятся базы данных с технических показателями средств для бурения скважин, информационно-поисковая подсистема для автоматизированного поиска и выбора текущих параметров. Техническое обеспечение для обработки, хранения и управления ТПБНС снабжено микропроцессорным модулем для обработки данных, поступающие из датчиков управления и контроля технологического процесса бурения нефтяной скважины [2]. Одним из технических средств управления и контроля в нефтяных скважинах является датчик измерения усилий, фиксирующий текущие данные нагрузки от штанги глубинного насоса, от надежности работы которой, зависит долговечность бурильного оборудования.

В этой связи, в рамках обеспечения требуемых нагрузок в штанге глубинного насоса бурильной установки, предлагается новая конструкция датчика по сравнению с магнитноупругим датчиком, который применяется в настоящее время в установках [10]. Данный датчик, конструктивно размещающийся в цилиндрическом кольце, имеет более простую структурную схему. Его работоспособность и надежность функционирования выше, чем существующие прототипы такого датчика [6]. Изготовленный проект датчика усилия с чувствительным цилиндрическим элементом для фиксирования нагрузок от штанги глубинного насоса в бурильной установке, представлен на рис. 2. 


\begin{tabular}{l|lrl|l|ll} 
& ISRA (India) & $=\mathbf{1 . 3 4 4}$ & SIS (USA) & $=\mathbf{0 . 9 1 2}$ & ICV (Poland) & $=\mathbf{6 . 6 3 0}$ \\
Impact Factor: & ISI (Dubai, UAE) $=\mathbf{0 . 8 2 9}$ & PUHU (Russia) $=\mathbf{0 . 1 7 9}$ & PIF (India) & $=\mathbf{1 . 9 4 0}$ \\
& GIF (Australia) & $\mathbf{0 . 5 6 4}$ & ESJI (KZ) & $=\mathbf{1 . 0 4 2}$ & & \\
& JIF & $=\mathbf{1 . 5 0 0}$ & SJIF (Morocco) $=\mathbf{2 . 0 3 1}$ & &
\end{tabular}

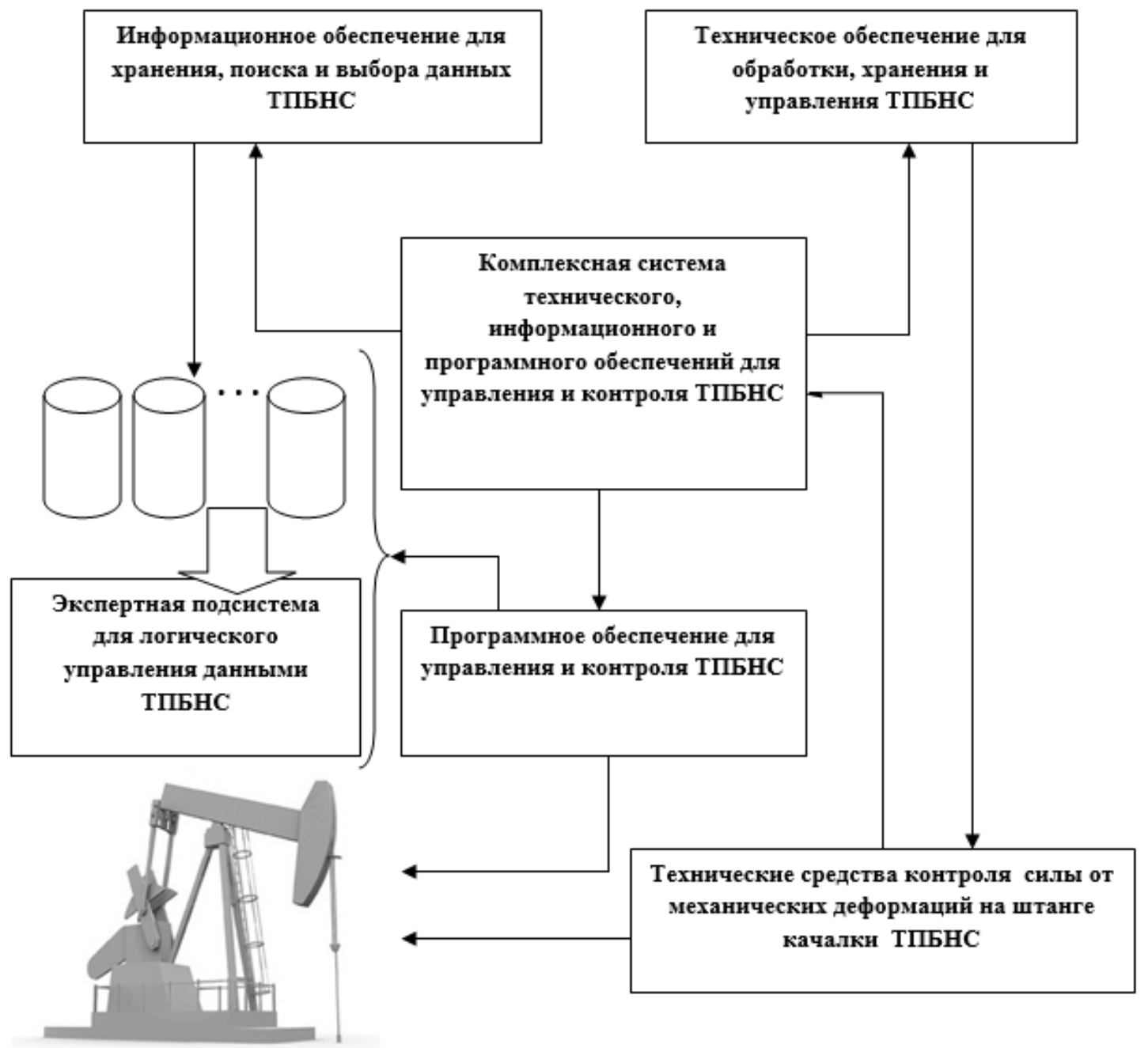

Рисунок 1 - Комплексная автоматизированная схема для управления и контроля технологического процесса бурения нефтяной скважины.

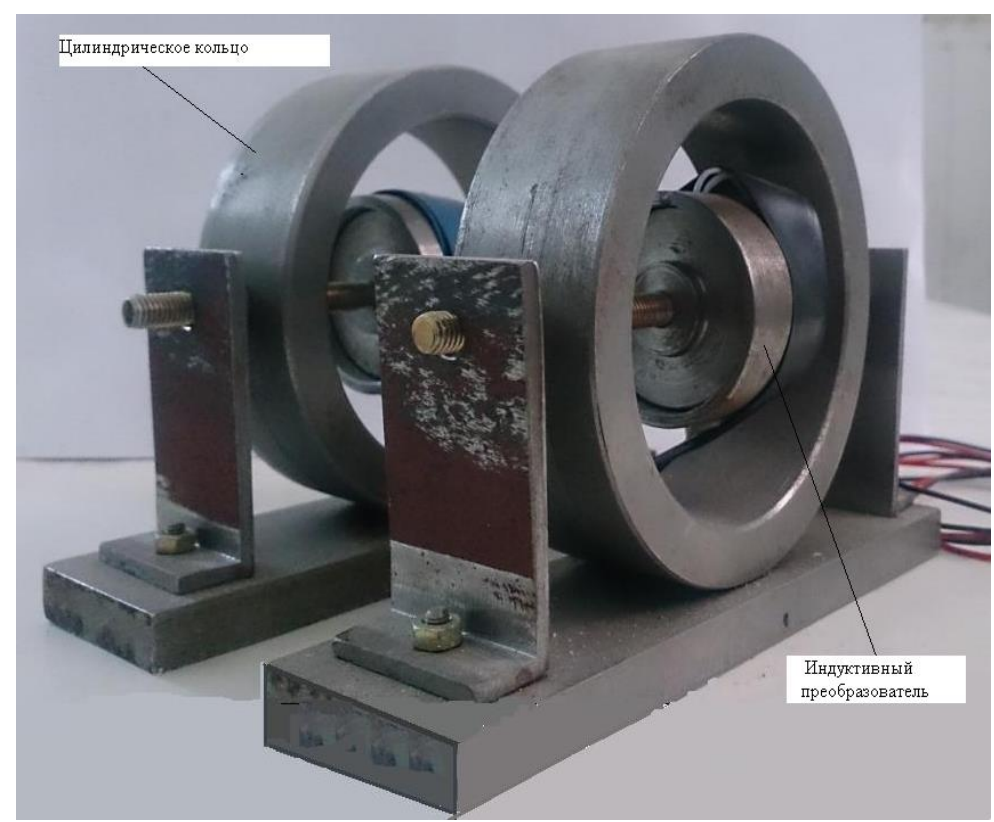

Рисунок 2 - Датчик усилия с чувствительным цилиндрическим элементом для фиксирования нагрузок от штанги глубинного насоса в бурильной установке. 


\begin{tabular}{|c|c|c|c|c|c|c|}
\hline Impact Factor: & $\begin{array}{l}\text { ISRA (India) } \\
\text { ISI (Dubai, UAF } \\
\text { GIF (Australia) } \\
\text { JIF }\end{array}$ & $\begin{array}{l}=1.344 \\
=0.829 \\
=0.564 \\
=1.500\end{array}$ & $\begin{array}{l}\text { SIS (USA) } \\
\text { PИНЦ (Russia) } \\
\text { ESJI (KZ) } \\
\text { SJIF (Morocco) }\end{array}$ & $\begin{array}{l}=0.912 \\
=0.179 \\
=1.042 \\
=2.031\end{array}$ & $\begin{array}{l}\text { ICV (Poland) } \\
\text { PIF (India) }\end{array}$ & $\begin{array}{l}=6.630 \\
=1.940\end{array}$ \\
\hline
\end{tabular}

Назначением спроектированного датчика с подключенным к нему чувствительным элементом является фиксирование текущих нагрузок от штанги глубинного насоса при нефтедобыче и своевременного извещения о состоянии технологического процесса скважины. При фиксировании отклонений нагрузок от стандартных норм принимается решение о стабилизации работы глубинного насоса в бурильной установке. Размещенный на оси чувствительного цилиндрического кольца [7], датчик состоит из неподвижного сердечника и двухобмоточной катушки [8]. При нагрузках штанги глубинного насоса, установленный между траверсами, датчик фиксирует деформации чувствительного цилиндрического кольца. Соответствующий текущий сигнал передается в микропроцессорный модуль, откуда обработанная информация передается в устройство контроля нефтедобывающей скважины, далее согласно опросу по радиочастотному каналу передается в центральный диспетчерский центр. Микропроцессорный модуль, чувствительный элемент с датчиком деформации, источник питания и модуль радиомодема размещены внутри неподвижного монолитного корпуса.

Разработанная интеллектуальная система обеспечивает преобразование входных аналоговых сигналов в числовые в микропроцессорном модуле. Для надежного функционирования системы в целом требуется выбор типа микропроцессора в зависимости от нагрузок штанги глубинного насоса при практическом функционировании нефтяной скважины.

Полученные результаты из расчета на усилие не должны превышать допустимых напряжений для разработанного толстостенного кольца в соответствии с выбранным материалом изготовления и его конструкционными размерами. Экспериментальный вариант чувствительного элемента датчика усилия рассчитан на $P=10000$ кг для штанги глубинного насоса. В зависимости от вышеуказанных требований, выбирается микропроцессор типа ATmega32-16. Структурная схема системы управления глубинного насоса нефтяной скважины представлена на рис. 3.

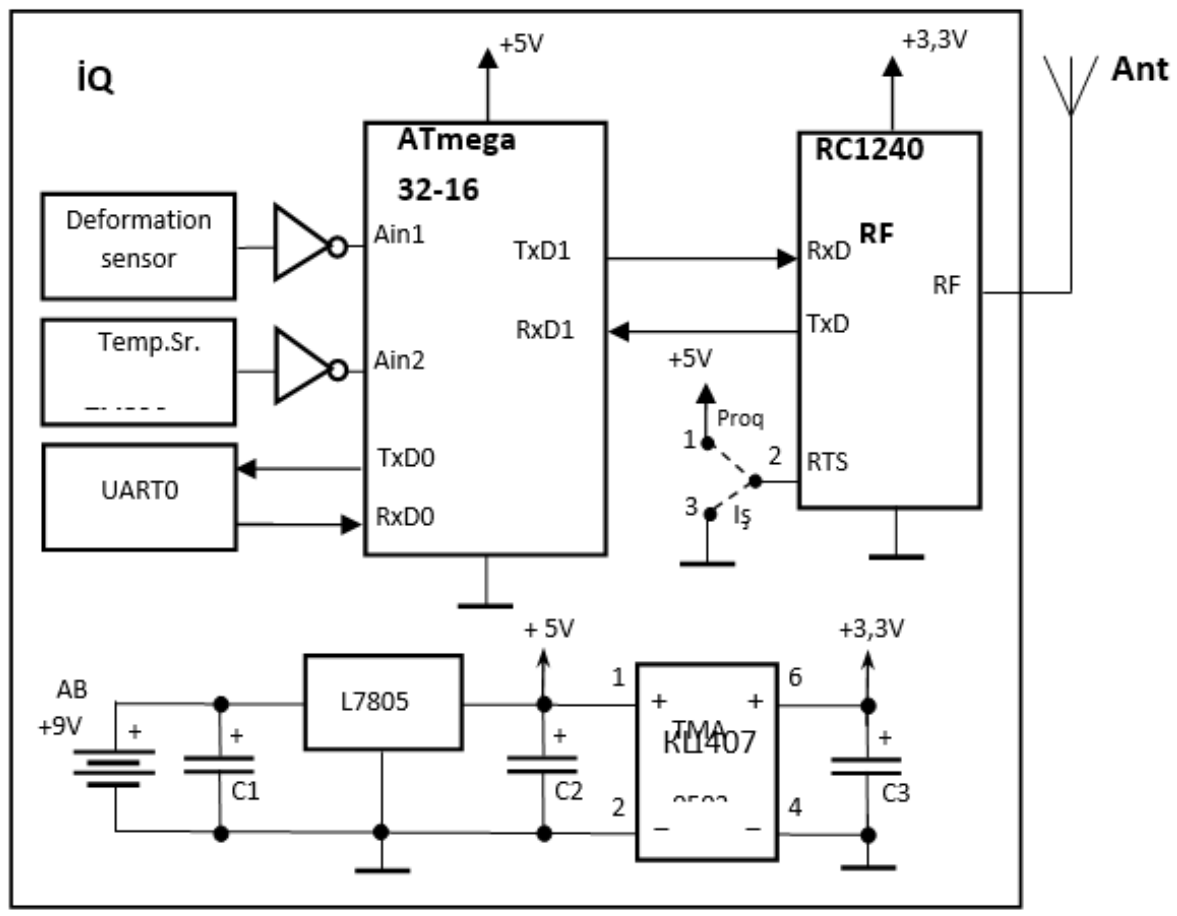

Рисунок 3 - Структурная схема системы управления глубинного насоса нефтяной скважины на основе микропроцессора ATmega32-16. 
Работа системы управления обеспечивается при воздействии на эластическую часть датчика деформации. При этом сопротивление датчика деформации изменяется, а на выходе датчика образуется напряжение. Для экспериментальных исследований с помощью показателей вольтметра проверяется показатель напряжения. Далее с помощью операционного усилителя для калибровки и вычисления показатель напряжения передается в измерительный прибор. Датчик деформации в данном испытании обеспечивает подачу аналогового сигнала в соответствии с измеряемой статистически или инерционно изменяющей силой сжатия и расширения.

\section{Результаты}

В рамках решения научноисследовательских задач в статье были получены следующие результаты:
1. Предложена комплексная автоматизированная схема для управления и контроля технологического процесса бурения нефтяных скважин (ТПБНС) на подуровнях технического, программного и информационного обеспечения;

2. Разработан датчик усилия с чувствительным цилиндрическим элементом для фиксирования нагрузок от штанги глубинного насоса в бурильной установке [10];

3. В зависимости от требований по выбору материала изготовления, конструкционных размеров и максимальных нагрузок штанги глубинного насоса выбирается микропроцессор типа ATmega32-16 и структурная схема системы управления нефтяной скважины.

\section{References:}

1. Aliev TA, Guluev GA, Rzaev AG, Aliev YG, Rizvanov MG (2011) Intellektualizirovannaya Stantsiya Upravleniya Stankom Kachalki, Zhurnal: "Mekhatronika, avtomatizatsiya, upravlenie". Moscow. Izdatel'stvo "Novye tekhnologii” №8, pp.17-20.

2. Gumerov RI (2009) Praktikum po mikroprotsessoram, Mikrokontrollery AVR, Kazan' -2009.

3. Isaev AS (2007) Ekonomika Neftyanoy i gazovoy promyshlennosti Azerbaydzhana, 2007.

4. Muradverdiev AS (2000) Neftyanaya promyshlennost' Azerbaydzhana $\mathrm{v}$ usloviyakh perekhoda $\mathrm{k}$ rynochnoy ekonomike - Baku, 2000.

5. Movsumzade AE, Alizade MF (2001) Pervye popytki ispol'zovaniya kontrol'noizmeritel'nykh priborov $\mathrm{v}$ neftyanom proizvodstve // Neftepererabotka i neftekhimiya. - 2001, №4.

6. Pershin VF, Selivanov YT (2002) Raschet na prochnost' tonkostennykh obolochek vrashcheniya i tolstostennykh tsilindrov. Metodicheskoe posobie. Tambov: Izd.-vo, TGTU, 2002 - 20 p.

7. Belyaev NM, Sinitskiy AK (1938) Napryazhenie i deformatsii $\mathrm{v}$ tolstostennykh tsilindrakh pri uprugoplasticheskom sostoyanii materiala. «Izvestiya otdeleniya tekhnicheskikh nauk Akademii Nauk SSSR» 1938.

8. Ginzburg VB (1970) «Magnitouprugie datchiki», izd. «Energiya», Moscow, 1970, 72p.

9. Gorbatikov VA, Ryskin MN (1965) Proektirovanie kompleksnoy avtomatizatsii neftyanykh promyslov. Izdatel'stvo «Nedra», Moscow, 1965.

10. Mammadov FI, Mammadov CF, Rasulov HR, Rzayev AH, Aliyev YH (2015) ilindrık halqanin deformasiyası hesabına islayan quvva vericisinin tadqiqi. Transaction of Azerbaijan National Academy of Sciences, Series of Physical-Technical and Mathematical Sciences: Informatics and Control Problems, Vol. XXXV, No.3, 2015. 